\title{
INTEROPERABILITY IN NHS HOSPITALS MUST BE IMPROVED: THE CQC SHOULD BE A KEY ACTOR IN THIS PROCESS
}

"NHS Interoperability and the CQC"

Abstract

For the better part of this century, the UK National Health Service (NHS) has aimed to transform itself into a digital service. Interoperability refers to the capability of multiple Electronic Medical Record (EMR) systems to exchange and make use of information cooperatively and forms a key part of this digital transformation. Development of interoperability is limited by both lack of incentive for hospital Trusts, and the reluctance of EMR vendors to implement interoperable systems. We discuss the role that expanded interoperability would play in the NHS, and reasons for the lack of progress to date. Within a digital health service, interoperability is a key component of safety and quality. We argue that it is essential for the Care Quality Commission (CQC), as an independent national healthcare regulator, to monitor interoperability in NHS hospitals. While the CQC has no power over vendors themselves, such regulation will incentivize Trusts to seek solutions. CQC involvement can accelerate progress and enable downstream benefits for the health service as a whole. 


\section{Introduction}

The transformation of the National Health Service (NHS) into a digital service has been slower than other comparable healthcare systems [1]. Seventeen years on from the National Programme for IT, interoperability between NHS hospital Trusts remains highly variable across the country. In order to accelerate progress, we argue that the Care Quality Commission (CQC) should provide regulatory oversight.

Here we set out three reasons for the CQC to take on this role. First, regulation of this area falls under the CQC's remit since the ability for care providers to exchange and access data is fundamental to both patient safety and quality of care. Second, a regulatory requirement can force the prioritisation of interoperability, and encourage Trusts to seek solutions from electronic medical record (EMR) vendors. Third, interoperability has the potential to benefit the CQC itself, through data driven identification of patient safety problems.

Data sharing is necessary to realise the long-term vision of the NHS

Interoperability is - today - integral to safe and high-quality care [2] [3]. It describes the ability of disparate electronic systems to communicate with each other and exchange data, and the ability for such data to be accessed and used cooperatively [4]. In healthcare systems that make significant use of IT, considerable numbers of patient safety incidents and adverse drug events have been linked to interoperability related challenges [5] [6]. Plans to improve interoperability have been part of multiple recent NHS documents, including the NHS Long 
Term Plan [7], the Wachter Review [8], the Topol Review [9], and the Five Year Forward View [10].

The end-benefits of advanced interoperability are well recognised. As well as reducing the administrative burden of gathering data for clinicians, interoperability is vital to unlocking the potential of the government's population health management vision [11]. Interoperability allows transformation of fragmented data assets into well curated research datasets [12]. Furthermore, advances in data sharing and access could eliminate the process of manual data collection for NHS audit.

To date, progress in hospital interoperability in the UK has been limited

Progress on interoperability has been slow. In the 2016 NHS Digital Maturity survey, only 56 out of 249 surveyed Trusts shared care records digitally with non-primary care providers. More recently, an independent survey on NHS interoperability showed that $33 \%$ of surveyed hospital Trusts could not electronically access outside patient data [13].

The principle challenge in delivering interoperability is the fragmentation of responsibility for IT infrastructure. Multiple vendors are contracted to create proprietary EMRs tailored for local trusts with little thought to external data sharing. To allow for useful interoperability in this environment, both standardisation of data between EMRs, and access to a wide scope of data, must be implemented. 
Policymakers have attempted to tackle this issue [14] but there is little incentive for vendors to make changes in the scope that is required. More needs to be done, with challenging but achievable timelines set nationally and implemented locally under regulatory pressure.

Regulating interoperability clearly falls within the CQC's stated remit

There is now a clear obligation for the CQC to use their regulatory powers to monitor interoperability for three reasons - clinical safety, quality of care, and to provide an independent voice on the state of digital performance in NHS hospitals.

Firstly - interoperability between NHS hospitals impacts on safe care, and thus falls under the remit of the CQC. The CQC already implements this reasoning in their inspection frameworks for ambulance [15], emergency [16] care and acute [17] hospital services. Under the umbrella of "Safety", lines of enquiry ask about information availability, coordination between information systems and data access (S3.4). The CQC has previously evaluated ambulance Trusts in the above areas. These aspects are no less important to patient safety in hospitals, and should now become a key part in routine CQC reports for NHS hospital Trusts.

Second, the CQC already subscribes to the model of interoperability as a key quality indicator. As we have described, the downstream benefits of interoperability are recognised, and are laid out in key NHS policy documents of the past few years. The CQC have launched their own 'Integrations, pathways, and place' programme to "look at how well services work together" and "how the way we regulate could respond to new ways of providing care" [18]. Policymakers have opened the door for regulation: Personalised Health and Care 2020 states 
that the CQC should take digital services into account from 2016 as part of its regulatory regime.

Third, an independent voice is needed to provide transparency to the progress of hospital interoperability, and related factors such as trust-vendor relationships. The best current data on our national progress in NHS interoperability comes from a one-off survey by an American consultancy [13]. Regular independent assessment, from an experienced regulator at a trust level, will demonstrate how we are progressing along our digital roadmap. A stated aim of the CQC is to provide an independent voice on "the state of health and adult social care in England on issues that matter to the public, providers and stakeholders" [19]. This voice can be used to empower change and understanding of the importance of sharing information.

Practically, the CQC can be an effective regulator

The introduction of CQC regulation can result in the expansion of interoperability in NHS hospitals. Without initiative from vendors to improve interoperability, the onus must be on Trusts themselves - CQC regulation provides Trusts with substantial incentive and penalty to look for solutions.

Regulatory weight will force Trusts to prioritise implementation of interoperability, and thereby indirectly encourage vendors to provide solutions. The current relationship between Trusts and vendors is highly asymmetrical: EMR systems are in the control of software 
vendors who generally charge monetary fees for additional features. Currently, this may discourage Trusts from prioritising upgrades towards interoperable systems. However - a regulatory requirement would turn interoperability into a key prerequisite that is prioritised in any contract negotiations or discussion about EMR updates. Data collected [20] has shown that very few acute NHS Trusts currently consider franchising when contracting EMRs. Armed with a regulatory requirement, Trusts can present a united and systematic approach to negotiating for interoperability, resulting in preferential consideration being given to vendors that offer more expansive interoperability as part of their EMR package. This possibility of balancing vendor-provider asymmetries is particularly true for smaller companies producing specialist software within single specialties, or for use with specific procedures.

Finally, interoperability could usher a new era for arms-length body data collection and use in the NHS. At present, the majority of CQC assessment is conducted by visiting inspectors at a minimum of every five years. The benefit to the CQC includes early identification of worrying trends in patient safety, allowing for ad hoc inspection and earlier intervention.

The CQC may choose to incorporate assessment of interoperability capabilities into existing inspections with a checklist system usable by generalist inspectors. Trusts can be required to provide written submissions that are centrally assessed as part of its evaluation. Ultimately, training a specific cadre of inspectors would allow the development of separate inspection programmes to review Trust EMR implementation and interoperability in detail. A barrier to such proposals may be expense, although the cost of such measures would be variable and dependent on implementation, with central evaluation likely cheaper than the development of separate inspections. Given the opportunity costs of physician time alone, it is important to consider efficient use of health IT [21]. 


\section{Conclusion}

The involvement of the CQC can act as a catalyst to improve interoperability within the NHS. This form of regulation by the CQC could also act as an enabler of pooling customer power across NHS Trusts, thus rebalancing the market forces which are currently in favour of the EMR vendors. Ultimately, this could contribute to better procurements and more effective EMR systems in the NHS.

We recognise that the regulatory powers of the CQC begin and end in the quality, safety and transparency of patient care. While we have discussed the benefits of interoperability which extend beyond this remit, we do not propose that CQC has oversight or visibility of these downstream functionalities. Rather, regulating interoperability is an enabler for these functionalities to occur, particularly as it opens the door for systems level discussion with vendors and policy-makers. The CQC clearly has no regulatory power over vendors themselves, but the CQC is able to deem progress unsatisfactory and oblige the prioritisation of interoperability, as well as transparency, in trust-vendor discussions.

The role of the CQC proposed here will only be effective within the broader context of oversight from and cooperation with other NHS arms-length bodies. NHSX is a newly formed unit which could take a leadership role with regards to interoperability. Digital policy, digital standard setting and interoperability fall explicitly within the remit of NHSX. NHSX could therefore provide clear guidance to the CQC on outcomes and methodologies for regulation of interoperability. In addition, NHSX could coordinate action by other bodies on interoperability. For example, NHS England could set tariffs to ensure that there are payment incentives for providers to ensure interoperability within local health economies. The 
importance of the CQC's role in this framework is direct regulatory implication for NHS Trusts.

The NHS Long Term Plan, and several other recent policy documents embrace a digital future for the NHS. The setting up of digital health fellowships to train clinicians in database science, the appointment of Trusts as global digital exemplars, and regions as local health and care record exemplars are important steps towards developing and utilising interoperability in the future. Interoperability is a key enabler to realising wider ambitions but could rapidly become a roadblock to this vision without further work.

\section{Bibliography}

1 Matthew Honeyman, Phoebe Dunn, Helen McKenna. An introduction to the digital agenda and plans for implementation. The King's Fund 2016.

2 Kaelber DC, Bates DW. Health information exchange and patient safety. Journal of Biomedical Informatics 2007;40:S40-5. doi:10.1016/j.jbi.2007.08.011

3 Walker J, Pan E, Johnston D, et al. The Value Of Health Care Information Exchange And Interoperability: There is a business case to be made for spending money on a fully standardized nationwide system. Health Affairs 2005;24:W5-10-W5-18. doi:10.1377/hlthaff.W5.10

4 What is Interoperability? HIMSS. 2016.http://www.himss.org/library/interoperabilitystandards/what-is-interoperability (accessed 1 Jun 2017). 
5 Adams K, Howe J, Fong A, et al. An Analysis of Patient Safety Incident Reports Associated with Electronic Health Record Interoperability. Appl Clin Inform. 2017;08(02):593-602. doi:10.4338/ACI-2017-01-RA-0014

6 Kaelber DC, Bates DW. Health information exchange and patient safety. Journal of Biomedical Informatics. 2007;40(6):S40-S45. doi:10.1016/j.jbi.2007.08.011

7 NHS Long Term Plan. https://www.longtermplan.nhs.uk/ (accessed 12 Apr 2019).

8 Wachter - Making IT Work Harnessing the Power of Health Inf.pdf. https://assets.publishing.service.gov.uk/government/uploads/system/uploads/attachment_ data/file/550866/Wachter_Review_Accessible.pdf (accessed 12 Apr 2019).

9 Topol review. Health Education England. 2018.https://www.hee.nhs.uk/our-work/topolreview (accessed 12 Apr 2019).

10 NHS England. Five Year Forward View. 2014. https://www.england.nhs.uk/wpcontent/uploads/2014/10/5yfv-web.pdf

11 Home. Healthcare Gateway. http://healthcaregateway.co.uk/ (accessed 31 May 2017).

12 Harris S, Shi S, Brealey D et al. Critical Care Health Informatics Collaborative (CCHIC): Data, tools and methods for reproducible research: A multi-centre UK intensive care database. Int J Med Inform 2018 Apr;112:82-89. doi: 10.1016/j.ijmedinf.2018.01.006

13 NHS Interoperability 2018. Klas Research 2018.

14 Are NHS England still requiring Trusts to achieve the October and December 2018 deadlines for ToC and Care Connect? - INTEROPen. 
https://www.interopen.org/ufaqs/are-nhs-england-still-requiring-trusts-to-achieve-theoctober-and-december-2018-deadlines-for-toc-and-care-connect/ (accessed 12 Apr 2019).

15 Care Quality Commission - Ambulance Inspection Framework. https://www.cqc.org.uk/sites/default/files/20190122_NHS_ambulance_Resilience_inspec tion_framework_v3.pdf

16 Care Quality Commission - Emergency Inspection Framework. https://www.cqc.org.uk/sites/default/files/20190122_Ambulance_E\%26UC_Core_Servic e_Framework_for_NHS_and_IH_Providers_v4.pdf

17 Care Quality Commission - Urgent Care Inspection Framework. https://www.cqc.org.uk/sites/default/files/20190122_Core_Services_framework_urgent_ and_emergency_care_for_NHS_and_IH_services_v7.pdf

18 Care Quality Commission - Coordinated Care. https://www.cqc.org.uk/what-wedo/coordinated-care/coordinated-care

19 Care Quality Commission - "Our Current Mode of Regulation". https://www.cqc.org.uk/sites/default/files/20171114_exe_procurementinfo.pdf

20 Budhdeo S et al. 2019. Interoperability in the NHS - a Situation Analysis by Freedom of Information Requests. Unpublished Manuscript.

21 Hilton J. 2019. Careless costs related to inefficient technology used within NHS England. Clinical Medicine. 2019;Nov 8. pii: clinmed.2019-0340. doi: 10.7861/clinmed.2019-0340 